OPEN ACCESS

Edited by:

Dong-Gyu Jo,

Sungkyunkwan University,

South Korea

Reviewed by:

Minho Moon,

Konyang University, South Korea

Nam Chul Kim,

University of Minnesota Twin Cities,

United States

${ }^{*}$ Correspondence:

Yong LuO

luoyongtt@163.com

${ }^{\dagger}$ These authors have contributed equally to this work

Received: 06 October 2019 Accepted: 04 February 2020

Published: 28 February 2020

Citation:

Huang W, Zhou Y, Tu L, Ba Z, Huang $J$, Huang $N$ and Luo $Y$ (2020) TDP-43: From Alzheimer's

Disease to Limbic-Predominant Age-Related TDP-43

Encephalopathy.

Front. Mol. Neurosci. 13:26. doi: 10.3389/fnmol.2020.00026

\section{TDP-43: From Alzheimer's Disease to Limbic-Predominant Age-Related TDP-43 Encephalopathy}

\author{
Wendi Huang ${ }^{1 \dagger}$, Yongjian $\mathrm{Zhou}^{2 \dagger}$, Lin $\mathrm{Tu}^{3}$, Zhisheng $\mathrm{Ba}^{3}$, Juan Huang ${ }^{4}$, Nanqu Huang ${ }^{3}$ \\ and Yong $\mathrm{LuO}^{3 *}$
}

${ }^{1}$ Department of Pediatrics, Guizhou Medical University, Guizhou, China, ${ }^{2}$ School of Graduate Studies, Zunyi Medical University, Guizhou, China, ${ }^{3}$ The Third Affiliated Hospital of Zunyi Medical University, The First People's Hospital of Zunyi, Guizhou, China, ${ }^{4}$ School of Public Health, Zunyi Medical University, Guizhou, China

Since the discovery of TAR DNA-binding protein 43 (TDP-43) in 1995, our understanding of its role continues to expand as research progresses. In particular, its role in the pathogenesis of Alzheimer's disease (AD) has drawn increasing interest in recent years. TDP-43 may participate in various pathogenic mechanisms underlying $A D$, such as amyloid $\beta$ deposition, tau hyperphosphorylation, mitochondrial dysfunction, and neuroinflammation. Because $A D$ is complex and heterogeneous, and because of the distinct characteristics of TDP-43, mostly seen in the oldest-old and those with more severe clinical phenotype, subcategorization based on specific features or biomarkers may significantly improve diagnosis and treatment. AD-like cognitive dysfunction associated with TDP-43 pathology may therefore be a distinct encephalopathy, referred to as limbic-predominant age-related TDP-43 encephalopathy (LATE).

Keywords: Alzheimer's disease, TDP-43, cognitive dysfunction, LATE, LATE-NC

\section{INTRODUCTION}

TAR DNA-binding protein 43 (TDP-43) is a 414 -amino-acid protein encoded by the TAR DNA binding protein gene on chromosome $1 \mathrm{p} 36$ (Buratti and Baralle, 2008). TDP-43 plays an important role in the regulation of RNA splicing, stability, transcriptional repression, and other cellular functions (Buratti and Baralle, 2001; Wang et al., 2004; Strong et al., 2007; Lee et al., 2011; Buratti, 2018). Since the discovery of TDP-43 in Ou et al. (1995), our understanding of its role continues to expand. In particular, its role in the pathogenesis of Alzheimer's disease (AD) has drawn increasing interest in recent years. TDP- 43 has been shown to be closely related to the onset and development of AD (Mercado et al., 2005; Vanden Broeck et al., 2014; Budini et al., 2017). Briefly, on the one hand, TDP-43 is related to neurotoxicity caused by its increased pathological aggregation, which is in turn caused by the aberrant phase transitions of TDP-43, induced by abnormal interactions between low-complexity domains (Mann et al., 2019). On the other hand, its role in AD is related to its loss of normal function in the central nervous system (CNS; Vanden Broeck et al., 2014; Budini et al., 2017). Loss of TDP-43 function promotes the use of cryptic splice sites, resulting in incorrect mRNA splicing and disease onset (Mercado et al., 2005). However, AD-like cognitive dysfunction with TDP-43 as the main marker may be defined as a new encephalopathy, referred to as limbicpredominant age-related TDP-43 encephalopathy (LATE; Nelson et al., 2019). 


\section{PATHOLOGICAL FEATURES OF TDP-43 IN AD}

$\mathrm{AD}$ is a progressive neurodegenerative disease, common in elderly individuals, and the most common form of dementia worldwide (Alzheimer's Disease International, 2018). Amyloid $\beta$ ( $\mathrm{A} \beta$ ) deposition is considered a major cause of $\mathrm{AD}$ pathogenesis (Kametani and Hasegawa, 2018). Although Biogen has submitted new analytical data for aducanumab to the Food and Drug Administration (FDA), solanezumab, bapineuzumab, and gantenerumab have not been shown to significantly improve cognitive function (Doody et al., 2014; de la Torre, 2014; Hung and $\mathrm{Fu}, 2017)$. Therefore, the $\mathrm{A} \beta$ cascade hypothesis is still under debate. Although conditional permission for GV-971 has been granted in China (Wang et al., 2019), neither the tau protein aggregation inhibitor LMTX (Adams, 2016), nor the 5-HT6 receptor antagonist idalopirdine (Atri et al., 2018) have shown therapeutic value for AD. Existing clinical trials are mostly aimed at patients with mild to moderate $\mathrm{AD}$, and TDP-43 pathology is mostly seen in the oldest-old and those with a more severe clinical phenotype (Wilson et al., 2011; Robinson et al., 2014; Nelson et al., 2019); between $20 \%$ and $50 \%$ of AD cases, and $75 \%$ of severe cases exhibit pathophysiological TDP-43 (AmadorOrtiz et al., 2007; Uryu et al., 2008). Therefore, TDP-43 may be a potential target for the treatment of severe $\mathrm{AD}$ with the TDP-43 pathology.

In addition to age and severity, the pathological features of TDP-43 in AD are also closely related to the area of distribution. Pathological TDP-43 aggregates have been observed in patients with frontotemporal lobe degeneration (FTLD) and amyotrophic lateral sclerosis (ALS). Similar findings were found in $\mathrm{AD}$ : pathological TDP-43 aggregation is more common in the limbic system of patients with $\mathrm{AD}$, including the hippocampus, amygdala, and adjacent cortex (Uryu et al., 2008). Based on FTLD tissue immunology research, pathological TDP-43 can be divided into four basic subtypes. Type A includes large numbers of neuronal cytoplasmic inclusions (NCI) and dystrophic neurites (DN). Type B includes large numbers of NCI and a small number of DN. Type $\mathrm{C}$ includes large numbers of longer and thicker DN than the A type, and also includes a small number of NCI. Type D has a large number of neuronal intranuclear inclusions (NII) and DN, and a small number of NCI (Mackenzie et al., 2011; Arai, 2014). In the cerebral cortex of patients with $\mathrm{AD}$ and dementia with Lewy bodies (DLB), type $\mathrm{A}$ is the most common pathological type (Josephs et al., 2016). Josephs et al. (2016) updated the TDP-43 in AD staging to six stages: (1) amygdala; (2) subiculum and entorhinal cortex; (3) dentate gyrus of the hippocampus and occipitotemporal cortex; (4) insular cortex, basal forebrain, ventral striatum, and inferior temporal cortex; (5) substantia nigra, midbrain tectum, and inferior olive; (6) middle frontal cortex and basal ganglia (Josephs et al., 2016). Of particular interest is the distribution of TDP-43 at these stages (especially as many of the areas involved are part of the limbic system), which can be understood as a result of TDP-43 spreading from neurons to neurons in a “prion-like" manner (Nonaka et al., 2013; Josephs et al., 2016). These studies showed that classification and determination of the location of pathological changes to TDP-43 may improve $\mathrm{AD}$ diagnosis.

\section{TDP-43 AND COGNITIVE DYSFUNCTION}

Abnormalities in TDP-43 correlate with worsened cognition and neurodegeneration (Laclair et al., 2016). Abnormal phosphorylation of TDP-43 in patients with $\mathrm{AD}$ was more pronounced in those with no cognitive impairment and mild cognitive impairment (MCI; Tremblay et al., 2011). Among subjects with no cognitive dysfunction, but with pathological changes in the hippocampus, $6 \%$ showed pathological changes in TDP-43. Furthermore, $42 \%$ of subjects with severe AD showed pathological changes in TDP-43 in the hippocampus (Rauramaa et al., 2011). Patients with pathological TDP-43 showed more severe hippocampal atrophy (Josephs et al., 2017) and worse performance on the Mini-Mental State Examination (MMSE), which suggested that pathological TDP-43 was highly associated with clinical signs in AD patients (Josephs et al., 2014). Multivariate logistic regression adjusted for age at death demonstrated that hippocampal sclerosis (HS) was the only pathologic predictor of abnormal TDP-43 immunoreactivity (Josephs et al., 2008). In addition, TDP43-positive subjects were more likely to exhibit cognitive dysfunction at the time of death than were TDP-43-negative subjects (Josephs et al., 2008). Patients with pathological changes in TDP-43 are more likely to show indifference, loss of compassion, stereotyped behavior, and action disorder (Jung et al., 2014). In addition to this clinical evidence, wild-type TDP-43 (wtTDP-43) expression was shown to cause hippocampal-dependent cognitive dysfunction in the CAMKII $\alpha$-tTA mouse model overexpressing human wtTDP-43 by selective CA2 subfield degeneration (Quadri et al., 2020). Although the detailed mechanisms have not been characterized, these data suggest that pathological TDP-43 may cause cognitive dysfunction.

\section{THE ROLE OF TDP-43 IN NEURONAL AND SYNAPTIC LOSS}

Neuronal loss and synaptic degeneration underlie AD pathogenesis, and represent common changes observed in many diseases associated with dementia (Jackson et al., 2019). Analysis of the role of TDP-43 in neuronal loss in the Cambridge city over-75 s cohort study revealed that TDP-43 inclusions were more common in the individuals who died later ( $>90$ years), or with clinical dementia. TDP-43 neuronal inclusions appear to be colocalized with severe neuronal loss (Keage et al., 2014). In HS characterized by hippocampal CA1 neuronal loss, only TDP-43 pathology increased the odds of HS (Nag et al., 2015). In addition to those clinical evidences, recent studies have shown that in the $5 \times$ FAD transgenic mice, chronic administration of PM1 (TDP43 mitochondrial localization inhibitory peptide) significantly reduced neuronal loss (Gao et al., 2020). This finding indicates that mitochondria-associated TDP-43 is likely involved in aspects of $\mathrm{AD}$ pathogenesis, especially neuronal loss. In the CAMKII $\alpha$-tTA mouse model overexpressing human wtTDP-43 
and $\alpha$-synuclein, overexpression of wtTDP-43 contributed to hippocampal CA2-specific pyramidal neuronal loss (Quadri et al., 2020). In another study examining the role of TDP-43 in synaptic loss, TDP-43 and perforant pathway synaptic loss were found to be the major contributors to dementia in the oldest-old (Robinson et al., 2014), and a trend between pTDP-43 pathology and synaptic loss in the frontal cortex of ALS cases was observed (Henstridge et al., 2018). In addition to these findings, the microglia-specific TDP-43 inducible conditional knockout (KO) mouse has been found to display drastic synaptic loss, suggesting the critical role of the loss of normal function of TDP-43 in this process (Paolicelli et al., 2017). Therefore, it is evident that TDP-43 plays a key role in neuronal and synaptic loss.

\section{THE ROLE OF TDP-43 IN THE PATHOGENESIS OF AD}

TDP-43 may be an important biomarker of AD. Some studies have evaluated the relationship of TDP-43 with $\mathrm{A} \beta$ deposition, tau hyperphosphorylation, mitochondrial dysfunction, and neuroinflammation (Herman et al., 2012; Laclair et al., 2016; Davis et al., 2017; Gao et al., 2020).

\section{TDP-43 and $A \beta$}

$\mathrm{A} \beta$, produced by hydrolysis of amyloid precursor protein (APP), is a major component of senile plaques and is considered to be a major pathological change in $\mathrm{AD}$ (Davis et al., 2017; Han et al., 2017). A $\beta$ deposition can lead to decreased solubility of TDP-43, resulting in abnormal aggregation and distribution of this protein (Xu et al., 2013; Chang et al., 2016). Studies have shown that pathological TDP-43 was found to be increased in the motor cortex of $A \beta_{1-42}$ lentivirus-transfected $3 \times$ Tg-AD mice, and $\mathrm{A} \beta$ clearance prevented increased TDP-43, which suggested that TDP-43 aggregation may be triggered by $A \beta$ (Herman et al., 2011). Cleavage of APP by $\beta$ secretase and $\gamma$-secretase can produce A $\beta$ and APP intracellular domain (AICD), respectively (Wang et al., 2014). Production of AICD has been shown to cause $\mathrm{AD}$, and its pathogenicity is independent of that of $A \beta$. Studies have shown that TDP-43 colocalizes with intranuclear AICD, resulting in up-regulation of p53 mRNA and exacerbation of AICD-induced apoptosis (Wang et al., 2014). Furthermore, TDP-43 depletion in forebrain neurons of an $\mathrm{AD}$ mouse model is correlated with increased prefibrillar oligomeric $\mathrm{A} \beta$ and decreased $\mathrm{A} \beta$ plaque burden, which exacerbates neurodegeneration, leading to cognitive and behavioral disorders (Laclair et al., 2016). Interestingly, Davis et al. (2017) showed that aberrantly phosphorylated TDP-43 and calcineurin interact. In a transgenic mouse overexpressing hippocampal and cortical neuronal TDP-43, reduced A $\beta$ plaque formation with increased TDP-43 was observed (Davis et al., 2017). The interactions between TDP-43 and $A \beta$ in $A D$ are unclear, and there is conflicting evidence within the literature. However, these findings suggest that TDP-43 may play an important role in $\mathrm{AD}$ and may be closely related to the regulation of $\mathrm{A} \beta$.

\section{TDP-43 and Tau}

Tau protein is the most abundant microtubule-associated protein. Microtubules, composed of tubulin and microtubuleassociated proteins, are components of the neuronal cytoskeleton and are essential for maintaining neuronal structure, neuronal plasticity, and axonal transport. However, hyperphosphorylation of tau protein in the brains of patients with $\mathrm{AD}$ results in formation of neurofibrillary tangles (NFTs), a significant contributor to disease (Gao et al., 2018). In patients with $\mathrm{AD}$, pathological TDP-43 has only been shown to aggregate in the limbic system, including the hippocampus, amygdala, and adjacent cortex (Uryu et al., 2008). Hyperphosphorylated tau protein also accumulates in these brain regions in patients with AD (Duyckaerts et al., 2009), suggesting that TDP-43 may colocalize with hyperphosphorylated tau protein. In mice with selective TDP-43 overexpression in an APP/PSEN1 background, TDP-43 increases abnormal tau aggregation, which may implicate TDP-43 expression in pre-tangle formation (Davis et al., 2017). However, studies have shown that TDP-43 does not regulate tau expression or splicing in $\mathrm{AD}$, which indicates that the mechanism by which TDP-43 contributes to AD may not be related to tau (Duyckaerts et al., 2009). Future studies should evaluate interactions between TDP-43 and tau pathology.

\section{TDP-43 and Mitochondrial Dysfunction}

Mitochondria are organelles that can replicate independently in a variety of eukaryotic cells. They provide energy and also participate in nearly all types of cell death, including apoptosis and necrosis, and contribute to a number of important physiological functions (Kroemer et al., 1998). Studies have shown that TDP-43 plays an important role in stabilizing mitochondrial function, and pathological TDP-43 can cause mitochondrial dysfunction (Izumikawa et al., 2017). Abnormal TDP-43 may cause mitochondrial dysfunction by affecting mitochondrial morphology, reactive oxygen species (ROS) generation, oxidative respiratory chain and localization. (1) Mitochondrial morphology: a significant reduction in mitochondrial cristae was observed in mouse neurons transfected with pathological TDP-43 (Yamashita and Kwak, 2014). The morphology of mitochondrial cristae is critical to the assembly and stability of respiratory chain super complexes, and affects mitochondrial function (Cogliati et al., 2013, 2016). (2) ROS: TDP-43 has been shown to increase mitochondrial production of ROS. Mitochondria are the main site of ROS production (Dan Dunn et al., 2015), and excessive accumulation of ROS can damage mitochondria (Perier et al., 2005; Cozzolino et al., 2013; Dan Dunn et al., 2015). (3) Oxidative respiratory chain: TDP-43 can decrease mitochondrial oxidative respiratory chain complex I and IV activity, dissipate the mitochondrial transmembrane potential, and reduce mitochondrial ATP synthesis (Stoica et al., 2014; Stribl et al., 2014). (4) Localization: abnormal localization of TDP-43 to mitochondria may alter mitochondrial morphology, resulting in mitochondrial dysfunction and induction of $\mathrm{AD}$ (Gao et al., 2020). Inhibition of abnormal localization of mutated TDP-43 in the mitochondria has been shown to reverse motor and cognitive dysfunction (Wang et al., 2017; 
Gao et al., 2020), and to prevent TDP-43-induced neurotoxicity (Wang et al., 2016).

\section{TDP-43 and Neuroinflammation}

Neuroinflammation, characterized by microglial activation, astrocyte proliferation, and increased cytokine expression, is a key factor in the pathogenesis of $\mathrm{AD}$. While acute inflammation protects the nervous system, chronic inflammation can contribute to AD development (Youmans and Wolozin, 2012; Huang et al., 2017). TDP-43 also plays an important role in the regulation of neuroinflammation. On the one hand, TDP-43 can enhance neuroinflammation by itself. Motor cortex of 2-months-old male Sprague-Dawley rats transfected with human wtTDP-43 exhibited increased expression of interleukin-6 (IL-6), tumor necrosis factor- $\alpha$ (TNF- $\alpha$ ), glial fibrillary acidic protein (GFAP), and other inflammatory markers (Herman et al., 2012). On the other hand, TDP-43 can cause excessive neuroinflammation through other factors; for example, the loss of the progranulin (PGRN) function can lead to abnormal aggregation of TDP-43, resulting in neuroinflammation and neuronal loss (Martens et al., 2012). Interestingly, when TDP-43 was conditionally $\mathrm{KO}$ in the microglial cells of $\mathrm{AD}$ mice, microglia showed strong phagocytosis, not only causing $A \beta$ clearing, but also causing synaptic loss (Paolicelli et al., 2017). These results suggest that the role of TDP-43 in AD is complex.

\section{IS IT TOO LATE TO DISCOVER LATE?}

Many studies have demonstrated the importance of TDP-43 in the pathogenesis of $\mathrm{AD}$ (Amador-Ortiz et al., 2007;
Josephs et al., 2008, 2016; Uryu et al., 2008). AD is a complex and heterogeneous disease, and a number of questions remain unresolved. Why do some individuals lose their memory first, while others experience loss of language or personality changes? Why do some individuals suffer from dementia at an early age, while others remain healthy until later in life? The heterogeneity of $\mathrm{AD}$ suggests that subcategorization of $\mathrm{AD}$ based on specific features or biomarkers may significantly improve diagnosis and treatment.

The AD-like cognitive dysfunction associated with TDP-43 pathology may represent a distinct encephalopathy because of its specific characteristics. Many researchers believe that the disease caused by TDP-43, characterized by changes in cognitive function, may be a novel disease: LATE (Nelson et al., 2019). Limbic-predominant age-related TDP-43 encephalopathy neuropathological change (LATE-NC) is defined by a stereotypical TDP-43 proteinopathy in elderly individuals, with or without coexisting HS (Nelson et al., 2019). A retrospective study found that LATE-NC was related to amnestic dementia syndrome that mimicked $\mathrm{AD}$ dementia (James et al., 2016). Moreover, LATE exhibits a different neuroanatomical distribution of FTLD, and is relatively more restricted than FTLD (Kadokura et al., 2009). Autopsy showed that at least $25 \%$ of identifiable cognitive dysfunctions were associated with LATE-NC, and many subjects with LATE-NC had $A \beta$ plaques, tauopathy, and a higher $\mathrm{p}$-tau burden (Latimer et al., 2019; Nelson et al., 2019). Diagnostic and staging guidelines for LATE-NC were proposed for use during routine autopsies. The protocol uses TDP-43 immunohistochemistry to reflect the organization of the amygdala, hippocampus, and middle

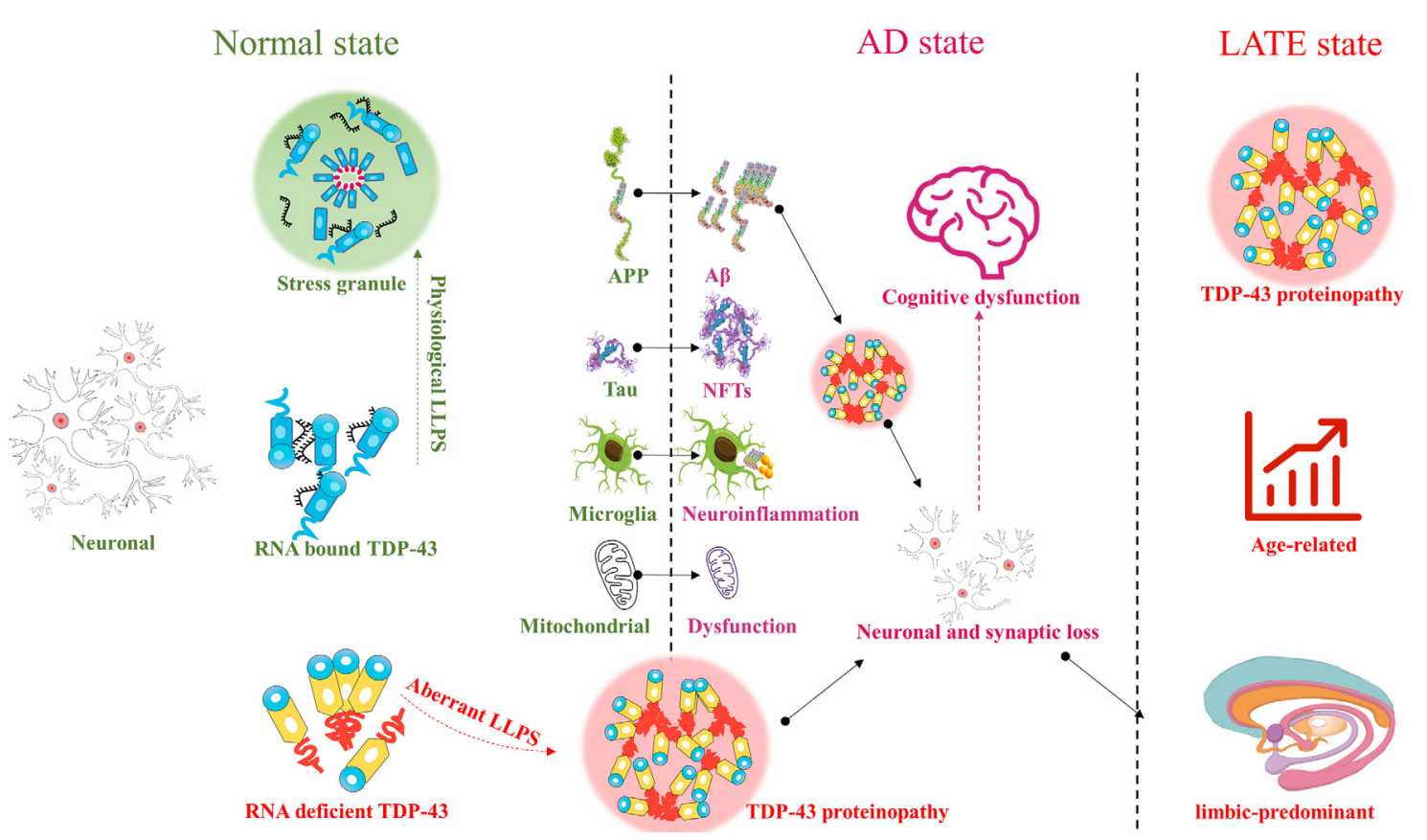

FIGURE 1 | TAR DNA-binding protein 43 (TDP-43) : from Alzheimer's disease (AD) to limbic-predominant age-related TDP-43 encephalopathy (LATE). 
frontal gyrus. Although LATE-NC preferentially affects the medial temporal lobe structure, other areas may also be affected (Nelson et al., 2019).

Since the shorthand for the disease is LATE, the interesting question that could be asked is: is it too late to discover LATE? Most clinical trials for treatment of $\mathrm{AD}$ have failed (Doody et al., 2014; de la Torre, 2014; Hung and Fu, 2017). This may have been due to the inclusion of many patients with LATE rather than those with pure AD. Most current inclusion/exclusion criteria are based on behavioral evaluations and neuroimaging, which may not distinguish between as-yet undefined subcategories of $\mathrm{AD}$ or distinct diseases. However, clinical trials are simply aimed at one of the several therapeutic targets for $\mathrm{AD}$, and though the treatment may be beneficial to some patients, a large number of ineffective results would be wasteful.

\section{CONCLUSIONS}

TDP-43 plays an important role in the CNS. Abnormal aggregation and localization of TDP-43 can cause mitochondrial dysfunction, aggravate neuroinflammation, and contribute to many diseases involving various regions of the brain. Abnormalities in TDP-43 in the upper and lower motor neurons can contribute to ALS, while abnormalities in TDP-43 in the frontal and temporal lobes can contribute to FTLD, and those in the limbic system to $\mathrm{AD}$. TDP-43 contributes to various pathogenic processes underlying $\mathrm{AD}$, such as $\mathrm{A} \beta$ deposition, tau hyperphosphorylation, mitochondrial dysfunction, and neuroinflammation. However, AD-like cognitive dysfunction associated with TDP-43 pathology appears to be distinct

\section{REFERENCES}

Adams, B. (2016). TauRx Misses Primary Endpoints in Alzheimer's Combo PhIII Trial [Online]. Available online at: https://www.fiercebiotech.com/biotech/taurx-misses-primary-endpointsalzheimer-s-combo-phiii-trial. Accessed July 27, 2016.

Alzheimer's Disease International. (2018). World Alzheimer Report 2018-The state of the Art of Dementia Research: New Frontiers [Online]. Available online at: https://www.alz.co.uk/research/WorldAlzheimerReport2018.pdf. Accessed September 21, 2018.

Amador-Ortiz, C., Lin, W. L., Ahmed, Z., Personett, D., Davies, P., Duara, R., et al. (2007). TDP-43 immunoreactivity in hippocampal sclerosis and Alzheimer's disease. Ann. Neurol. 61, 435-445. doi: 10.1002/ana.21154

Arai, T. (2014). Significance and limitation of the pathological classification of TDP-43 proteinopathy. Neuropathology 34, 578-588. doi: 10.1111/neup. 12138

Atri, A., Frölich, L., Ballard, C., Tariot, P. N., Molinuevo, J. L., Boneva, N., et al. (2018). Effect of idalopirdine as adjunct to cholinesterase inhibitors on change in cognition in patients with Alzheimer disease: three randomized clinical trials. JAMA 319, 130-142. doi: 10.1001/jama.2017.20373

Budini, M., Buratti, E., Morselli, E., and Criollo, A. (2017). Autophagy and its impact on neurodegenerative diseases: new roles for TDP-43 and C9orf72. Front. Mol. Neurosci. 10:170. doi: 10.3389/fnmol.2017.00170

Buratti, E. (2018). TDP-43 post-translational modifications in health and disease. Expert Opin. Ther. Targets 22, 279-293. doi: 10.1080/14728222.2018.1439923

Buratti, E., and Baralle, F. E. (2001). Characterization and functional implications of the RNA binding properties of nuclear factor TDP-43, a novel splicing regulator of CFTR exon 9. J. Biol. Chem. 276, 36337-36343. doi: 10.1074/jbc. $\mathrm{m} 104236200$ from that associated with $\mathrm{AD}$. Therefore, $\mathrm{AD}$-like cognitive dysfunction with TDP-43 as the main marker may be defined as a new encephalopathy, LATE, or may alternatively represent a subcategory of AD (Figure 1). Future studies should focus on redefining or emphasizing the important role of TDP-43 in cognitive dysfunction, whether as a subcategory of $\mathrm{AD}$, or as LATE, with the purpose of defining new diagnostic criteria or treatment strategies. It is never too late to discover TDP-43 and LATE.

\section{AUTHOR CONTRIBUTIONS}

$\mathrm{WH}$ and $\mathrm{YZ}$ contributed equally to this work and wrote the manuscript. $\mathrm{WH}, \mathrm{YZ}, \mathrm{LT}, \mathrm{ZB}, \mathrm{JH}, \mathrm{NH}$ and $\mathrm{YL}$ contributed to the critical revision of the manuscript and read and approved the submitted version.

\section{FUNDING}

This work was supported by the National Natural Science Foundation of China (81860710, 81650030); Guizhou Provincial Health and Health Commission (gzwjkj2019-1-064); Open Project Funding of Key Laboratory of Basic Pharmacology and Ministry of Education and Specialized National Medicine Ministry of Education International Cooperation Joint Laboratory (JCYL-K-012); Guizhou Province's Thousand-level Innovative Talents (2015-25); The 15851 Talent Elite Project; Guizhou Provincial Department of Science and Technology (2016-1165); The Science and Technology Joint Funds of Zunyi Science and Technology Bureau and The First People's Hospital of Zunyi (2017-29, 2018-160).

Buratti, E., and Baralle, F. E. (2008). Multiple roles of TDP-43 in gene expression, splicing regulation, and human disease. Front. Biosci. 13, 867-878. doi: $10.2741 / 2727$

Chang, X. L., Tan, M. S., Tan, L., and Yu, J. T. (2016). The role of TDP-43 in Alzheimer's disease. Mol. Neurobiol. 53, 3349-3359. doi: 10.1007/s12035-0159264-5

Cogliati, S., Enriquez, J. A., and Scorrano, L. (2016). Mitochondrial cristae: where beauty meets functionality. Trends Biochem. Sci. 41, 261-273. doi: 10.1016/j. tibs.2016.01.001

Cogliati, S., Frezza, C., Soriano, M. E., Varanita, T., Quintana-Cabrera, R., Corrado, M., et al. (2013). Mitochondrial cristae shape determines respiratory chain supercomplexes assembly and respiratory efficiency. Cell 155, 160-171. doi: 10.1016/j.cell.2013.08.032

Cozzolino, M., Ferri, A., Valle, C., and Carri, M. T. (2013). Mitochondria and ALS: implications from novel genes and pathways. Mol. Cell. Neurosci. 55, 44-49. doi: 10.1016/j.mcn.2012.06.001

Dan Dunn, J., Alvarez, L. A., Zhang, X., and Soldati, T. (2015). Reactive oxygen species and mitochondria: a nexus of cellular homeostasis. Redox Biol. 6, 472-485. doi: 10.1016/j.redox.2015.09.005

Davis, S. A., Gan, K. A., Dowell, J. A., Cairns, N. J., and Gitcho, M. A. (2017). TDP43 expression influences amyloidbeta plaque deposition and tau aggregation. Neurobiol. Dis. 103, 154-162. doi: 10.1016/j.nbd.2017.04.012

de la Torre, J. C. (2014). Phase 3 trials of solanezumab and bapineuzumab for Alzheimer's disease. N. Engl. J. Med. 370, 1459-1460. doi: 10.1056/nejmc1402193

Doody, R. S., Thomas, R. G., Farlow, M., Iwatsubo, T., Vellas, B., Joffe, S., et al. (2014). Phase 3 trials of solanezumab for mild-to-moderate Alzheimer's disease. N. Engl. J. Med. 370, 311-321. doi: 10.1056/NEJMoa 1312889 
Duyckaerts, C., Delatour, B., and Potier, M. C. (2009). Classification and basic pathology of Alzheimer disease. Acta Neuropathol. 118, 5-36. doi: 10.1007/s00401-009-0532-1

Gao, Y., Tan, L., Yu, J. T., and Tan, L. (2018). Tau in Alzheimer's disease: mechanisms and therapeutic strategies. Curr. Alzheimer Res. 15, 283-300. doi: 10.2174/1567205014666170417111859

Gao, J., Wang, L., Gao, C., Arakawa, H., Perry, G., and Wang, X. (2020). TDP43 inhibitory peptide alleviates neurodegeneration and memory loss in an APP transgenic mouse model for Alzheimer's disease. Biochim. Biophys. Acta Mol. Basis Dis. 1866:165580. doi: 10.1016/j.bbadis.2019.165580

Han, X. J., Hu, Y. Y., Yang, Z. J., Jiang, L. P., Shi, S. L., Li, Y. R., et al. (2017). Amyloid $\beta-42$ induces neuronal apoptosis by targeting mitochondria. Mol. Med. Rep. 16, 4521-4528. doi: 10.3892/mmr.2017.7203

Henstridge, C. M., Sideris, D. I., Carroll, E., Rotariu, S., Salomonsson, S., Tzioras, M., et al. (2018). Synapse loss in the prefrontal cortex is associated with cognitive decline in amyotrophic lateral sclerosis. Acta Neuropathol. 135, 213-226. doi: 10.1007/s00401-017-1797-4

Herman, A. M., Khandelwal, P. J., Rebeck, G. W., and Moussa, C. E. (2012). Wild type TDP-43 induces neuro-inflammation and alters APP metabolism in lentiviral gene transfer models. Exp. Neurol. 235, 297-305. doi: 10.1016/j. expneurol.2012.02.011

Herman, A. M., Khandelwal, P. J., Stanczyk, B. B., Rebeck, G. W., and Moussa, C. E. (2011). $\beta$-amyloid triggers ALS-associated TDP-43 pathology in AD models. Brain Res. 1386, 191-199. doi: 10.1016/j.brainres.2011.02.052

Huang, N. Q., Jin, H., Zhou, S. Y., Shi, J. S., and Jin, F. (2017). TLR4 is a link between diabetes and Alzheimer's disease. Behav. Brain Res. 316, 234-244. doi: 10.1016/j.bbr.2016.08.047

Hung, S. Y., and Fu, W. M. (2017). Drug candidates in clinical trials for Alzheimer's disease. J. Biomed. Sci. 24:47. doi: 10.1186/s12929-017-0355-7

Izumikawa, K., Nobe, Y., Yoshikawa, H., Ishikawa, H., Miura, Y., Nakayama, H., et al. (2017). TDP-43 stabilises the processing intermediates of mitochondrial transcripts. Sci. Rep. 7:7709. doi: 10.1038/s41598-017-06953-y

Jackson, J., Jambrina, E., Li, J., Marston, H., Menzies, F., Phillips, K., et al. (2019). Targeting the synapse in Alzheimer's disease. Front. Neurosci. 13:735. doi: 10.3389/fnins.2019.00735

James, B. D., Wilson, R. S., Boyle, P. A., Trojanowski, J. Q., Bennett, D. A., and Schneider, J. A. (2016). TDP-43 stage, mixed pathologies, and clinical Alzheimer's-type dementia. Brain 139, 2983-2993. doi: 10.1093/brain/aww224

Josephs, K. A., Dickson, D. W., Tosakulwong, N., Weigand, S. D., Murray, M. E., Petrucelli, L., et al. (2017). Rates of hippocampal atrophy and presence of post-mortem TDP-43 in patients with Alzheimer's disease: a longitudinal retrospective study. Lancet Neurol. 16, 917-924. doi: 10.1016/s14744422(17)30284-3

Josephs, K. A., Murray, M. E., Whitwell, J. L., Tosakulwong, N., Weigand, S. D., Petrucelli, L., et al. (2016). Updated TDP-43 in Alzheimer's disease staging scheme. Acta Neuropathol. 131, 571-585. doi: 10.1007/s00401-016-1537-1

Josephs, K. A., Whitwell, J. L., Knopman, D. S., Hu, W. T., Stroh, D. A., Baker, M., et al. (2008). Abnormal TDP-43 immunoreactivity in AD modifies clinicopathologic and radiologic phenotype. Neurology 70, 1850-1857. doi: 10.1212/01.wnl.0000304041.09418.b1

Josephs, K. A., Whitwell, J. L., Weigand, S. D., Murray, M. E., Tosakulwong, N., Liesinger, A. M., et al. (2014). TDP-43 is a key player in the clinical features associated with Alzheimer's disease. Acta Neuropathol. 127, 811-824. doi: 10.1007/s00401-014-1269-Z

Jung, Y., Dickson, D. W., Murray, M. E., Whitwell, J. L., Knopman, D. S., Boeve, B. F., et al. (2014). TDP-43 in Alzheimer's disease is not associated with clinical FTLD or Parkinsonism. Mov. Disord. 261, 1344-1348. doi: 10.1007/s00415-014-7352-5

Kadokura, A., Yamazaki, T., Lemere, C. A., Takatama, M., and Okamoto, K. (2009). Regional distribution of TDP-43 inclusions in Alzheimer disease (AD) brains: their relation to AD common pathology. Neuropathology 29, 566-573. doi: 10.1111/j.1440-1789.2009.01017.x

Kametani, F., and Hasegawa, M. (2018). Reconsideration of amyloid hypothesis and tau hypothesis in Alzheimer's disease. Front. Neurosci. 12:25. doi: 10.3389/fnins.2018.00025

Keage, H. A., Hunter, S., Matthews, F. E., Ince, P. G., Hodges, J., Hokkanen, S. R., et al. (2014). TDP-43 pathology in the population: prevalence and associations with dementia and age. J. Alzheimers Dis. 42, 641-650. doi: 10.3233/jad-132351
Kroemer, G., Dallaporta, B., and Resche-Rigon, M. (1998). The mitochondrial death/life regulator in apoptosis and necrosis. Annu. Rev. Physiol. 60, 619-642. doi: 10.1146/annurev.physiol.60.1.619

Laclair, K. D., Donde, A., Ling, J. P., Jeong, Y. H., Chhabra, R., Martin, L. J., et al. (2016). Depletion of TDP-43 decreases fibril and plaque $\beta$-amyloid and exacerbates neurodegeneration in an Alzheimer's mouse model. Acta Neuropathol. 132, 859-873. doi: 10.1007/s00401-016-1637-y

Latimer, C. S., Burke, B. T., Liachko, N. F., Currey, H. N., Kilgore, M. D., Gibbons, L. E., et al. (2019). Resistance and resilience to Alzheimer's disease pathology are associated with reduced cortical pTau and absence of limbicpredominant age-related TDP-43 encephalopathy in a community-based cohort. Acta Neuropathol. Commun. 7:91. doi: 10.1186/s40478-019-0743-1

Lee, E. B., Lee, V. M., and Trojanowski, J. Q. (2011). Gains or losses: molecular mechanisms of TDP43-mediated neurodegeneration. Nat. Rev. Neurosci. 13, 38-50. doi: 10.1038/nrn3121

Mackenzie, I. R., Neumann, M., Baborie, A., Sampathu, D. M., Du Plessis, D., Jaros, E., et al. (2011). A harmonized classification system for FTLD-TDP pathology. Acta Neuropathol. 122, 111-113. doi: 10.1007/s00401-0110845-8

Mann, J. R., Gleixner, A. M., Mauna, J. C., Gomes, E., Dechellis-Marks, M. R. Needham, P. G., et al. (2019). RNA binding antagonizes neurotoxic phase transitions of TDP-43. Neuron 102, 321.e8-338.e8. doi: 10.1016/j.neuron.2019. 01.048

Martens, L. H., Zhang, J., Barmada, S. J., Zhou, P., Kamiya, S., Sun, B., et al. (2012). Progranulin deficiency promotes neuroinflammation and neuron loss following toxin-induced injury. J. Clin. Invest. 122, 3955-3959. doi: $10.1172 /$ jci63113

Mercado, P. A., Ayala, Y. M., Romano, M., Buratti, E., and Baralle, F. E. (2005). Depletion of TDP 43 overrides the need for exonic and intronic splicing enhancers in the human ApoA-II gene. Nucleic Acids Res. 33, 6000-6010. doi: 10.1093/nar/gki897

Nag, S., Yu, L., Capuano, A. W., Wilson, R. S., Leurgans, S. E., Bennett, D. A., et al. (2015). Hippocampal sclerosis and TDP-43 pathology in aging and Alzheimer disease. Ann. Neurol. 77, 942-952. doi: 10.1002/ana.24388

Nelson, P. T., Dickson, D. W., Trojanowski, J. Q., Jack, C. R., Boyle, P. A., Arfanakis, K., et al. (2019). Limbic-predominant age-related TDP-43 encephalopathy (LATE): consensus working group report. Brain 142, 1503-1527. doi: 10.1093/brain/awz186

Nonaka, T., Masuda-Suzukake, M., Arai, T., Hasegawa, Y., Akatsu, H., Obi, T., et al. (2013). Prion-like properties of pathological TDP-43 aggregates from diseased brains. Cell Rep. 4, 124-134. doi: 10.1016/j.celrep.2013.06.007

Ou, S. H., Wu, F., Harrich, D., García-Martínez, L. F., and Gaynor, R. B. (1995). Cloning and characterization of a novel cellular protein, TDP-43, that binds to human immunodeficiency virus type 1 TAR DNA sequence motifs. J. Virol. 69, 3584-3596. doi: 10.1128/jvi.69.6.3584-3596.1995

Paolicelli, R. C., Jawaid, A., Henstridge, C. M., Valeri, A., Merlini, M., Robinson, J. L., et al. (2017). TDP-43 depletion in microglia promotes amyloid clearance but also induces synapse loss. Neuron 95, 297.e6-308.e6. doi: 10.1016/j.neuron.2017.05.037

Perier, C., Tieu, K., Guégan, C., Caspersen, C., Jackson-Lewis, V., Carelli, V., et al. (2005). Complex I deficiency primes Bax-dependent neuronal apoptosis through mitochondrial oxidative damage. Proc. Natl. Acad. Sci. U S A 102, 19126-19131. doi: 10.1073/pnas.0508215102

Quadri, Z., Johnson, N., Zamudio, F., Miller, A., Peters, M., Smeltzer, S., et al. (2020). Overexpression of human wtTDP-43 causes impairment in hippocampal plasticity and behavioral deficits in CAMKII-tTa transgenic mouse model. Mol. Cell. Neurosci. 102:103418. doi: 10.1016/j.mcn.2019. 103418

Rauramaa, T., Pikkarainen, M., Englund, E., Ince, P. G., Jellinger, K., Paetau, A., et al. (2011). TAR-DNA binding protein-43 and alterations in the hippocampus. J. Neural Transm. 118, 683-689. doi: 10.1007/s00702-0100574-5

Robinson, J. L., Molina-Porcel, L., Corrada, M. M., Raible, K., Lee, E. B., Lee, V. M., et al. (2014). Perforant path synaptic loss correlates with cognitive impairment and Alzheimer's disease in the oldest-old. Brain 137, 2578-2587. doi: 10.1093/brain/awu190

Stoica, R., De Vos, K. J., Paillusson, S., Mueller, S., Sancho, R. M., Lau, K. F., et al (2014). ER-mitochondria associations are regulated by the VAPB-PTPIP51 
interaction and are disrupted by ALS/FTD-associated TDP-43. Nat. Commun. 5:3996. doi: 10.1038/ncomms4996

Stribl, C., Samara, A., Trümbach, D., Peis, R., Neumann, M., Fuchs, H., et al. (2014). Mitochondrial dysfunction and decrease in body weight of a transgenic knock-in mouse model for TDP-43. J. Biol. Chem. 289, 10769-10784. doi: 10.1074/jbc.M113.515940

Strong, M. J., Volkening, K., Hammond, R., Yang, W., Strong, W., LeystraLantz, C., et al. (2007). TDP43 is a human low molecular weight neurofilament (hNFL) mRNA-binding protein. Mol. Cell. Neurosci. 35, 320-327. doi: 10.1016/j.mcn.2007.03.007

Tremblay, C., St-Amour, I., Schneider, J., Bennett, D. A., and Calon, F. (2011). Accumulation of transactive response DNA binding protein 43 in mild cognitive impairment and Alzheimer disease. J. Neuropathol. Exp. Neurol. 70, 788-798. doi: 10.1097/nen.0b013e31822c62cf

Uryu, K., Nakashima-Yasuda, H., Forman, M. S., Kwong, L. K., Clark, C. M., Grossman, M., et al. (2008). Concomitant TAR-DNA-binding protein 43 pathology is present in Alzheimer disease and corticobasal degeneration but not in other tauopathies. J. Neuropathol. Exp. Neurol. 67, 555-564. doi: 10.1097/nen.0b013e31817713b5

Vanden Broeck, L., Callaerts, P., and Dermaut, B. (2014). TDP-43-mediated neurodegeneration: towards a loss-of-function hypothesis? Trends Mol. Med. 20, 66-71. doi: 10.1016/j.molmed.2013.11.003

Wang, W., Arakawa, H., Wang, L., Okolo, O., Siedlak, S. L., Jiang, Y., et al. (2017). Motor-coordinative and cognitive dysfunction caused by mutant TDP-43 could be reversed by inhibiting its mitochondrial localization. Mol. Ther. 25, 127-139. doi: 10.1016/j.ymthe.2016.10.013

Wang, X., Sun, G., Feng, T., Zhang, J., Huang, X., Wang, T., et al. (2019). Sodium oligomannate therapeutically remodels gut microbiota and suppresses gut bacterial amino acids-shaped neuroinflammation to inhibit Alzheimer's disease progression. Cell Res. 29, 787-803. doi: 10.1038/s41422-0190216-X

Wang, J., Yan, K., Wu, Z. Q., Zheng, C. Y., Xu, R. X., Chen, L. H., et al. (2014). TDP-43 interaction with the intracellular domain of amyloid precursor protein induces p53-associated apoptosis. Neurosci. Lett. 569, 131-136. doi: 10.1016/j. neulet.2014.03.075

Wang, H. Y., Wang, I. F., Bose, J., and Shen, C. K. (2004). Structural diversity and functional implications of the eukaryotic TDP gene family. Genomics 83, 130-139. doi: 10.1016/s0888-7543(03)00214-3

Wang, W., Wang, L., Lu, J., Siedlak, S. L., Fujioka, H., Liang, J., et al. (2016). The inhibition of TDP-43 mitochondrial localization blocks its neuronal toxicity. Nat. Med. 22, 869-878. doi: 10.1038/nm.4130

Wilson, A. C., Dugger, B. N., Dickson, D. W., and Wang, D. S. (2011). TDP43 in aging and Alzheimer's disease-a review. Int. J. Clin. Exp. Pathol. 4, $147-155$.

Xu, G., Stevens, S. M. Jr., Moore, B. D., McClung, S., and Borchelt, D. R. (2013). Cytosolic proteins lose solubility as amyloid deposits in a transgenic mouse model of Alzheimer-type amyloidosis. Hum. Mol. Genet. 22, 2765-2774. doi: $10.1093 / \mathrm{hmg} / \mathrm{ddt} 121$

Yamashita, T., and Kwak, S. (2014). The molecular link between inefficient GluA2 Q/R site-RNA editing and TDP-43 pathology in motor neurons of sporadic amyotrophic lateral sclerosis patients. Brain Res. 1584, 28-38. doi: 10.1016/j.brainres.2013.12.011

Youmans, K. L., and Wolozin, B. (2012). TDP-43: a new player on the AD field? Exp. Neurol. 237, 90-95. doi: 10.1016/j.expneurol.2012.05.018

Conflict of Interest: The authors declare that the research was conducted in the absence of any commercial or financial relationships that could be construed as a potential conflict of interest.

Copyright (c) 2020 Huang, Zhou, Tu, Ba, Huang, Huang and Luo. This is an open-access article distributed under the terms of the Creative Commons Attribution License (CC BY). The use, distribution or reproduction in other forums is permitted, provided the original author(s) and the copyright owner(s) are credited and that the original publication in this journal is cited, in accordance with accepted academic practice. No use, distribution or reproduction is permitted which does not comply with these terms. 